\title{
Evaluation of Ivermectin as a Potential Treatment for Mild to Moderate COVID-19: A Double-Blind Randomized Placebo Controlled Trial in Eastern India
}

Ravikirti ${ }^{1}$, Ranjini Roy ${ }^{1}$, Chandrima Pattadar ${ }^{1}$, Rishav Raj ${ }^{1}$, Neeraj Agarwal ${ }^{2}$, Bijit Biswas ${ }^{3}$, Pramod Kumar Manjhi ${ }^{4}$ Deependra Kumar Rai ${ }^{5}$, Shyama ${ }^{1}$, Anjani Kumar ${ }^{1}$, Asim Sarfaraz ${ }^{6}$

\begin{abstract}
${ }^{1}$ Department of General Medicine, All India Institute of Medical Sciences, Patna, Bihar, India; ${ }^{2}$ Department of Community and Family Medicine, All India Institute of Medical Sciences, Bibinagar, Telangana, India; ${ }^{3}$ Department of Community and Family Medicine, All India Institute of Medical Sciences, Patna, Bihar, India; ${ }^{4}$ Department of Pharmacology, All India Institute of Medical Sciences, Patna, Bihar, India; ${ }^{5}$ Department of Pulmonary Medicine, All India Institute of Medical Sciences, Patna, Bihar, India; ${ }^{6}$ Department of Microbiology, All India Institute of Medical Sciences, Patna, Bihar, India
\end{abstract}

Corresponding author: Dr. Bijit Biswas, Department of Community and Family Medicine, All India Institute of Medical Sciences, Phulwarisharif, Patna: 801505, Bihar, India; email: drbijitbiswas@gmail.com

Received, June 6, 2021; Revised, July 6, 2021; Accepted, July 7, 2021; Published, July 15, 2021

\begin{abstract}
Background: There has been a growing interest in ivermectin ever since it was reported to have an in-vitro activity against severe acute respiratory syndrome coronavirus 2 (SARS-CoV-2). This trial was conducted to test the efficacy of ivermectin in mild and moderate coronavirus disease 19 (COVID-19). Methods: A double blind, parallel, randomised, placebo-controlled trial conducted among adult COVID-19 patients with mild to moderate disease severity on admission in a COVID dedicated tertiary healthcare of eastern India. Enrolment was done between $1^{\text {st }}$ August and $31^{\text {st }}$ October 2020. On day 1 and 2 post enrolment, patients in the intervention arm received ivermectin $12 \mathrm{mg}$ while the patients in the non-interventional arm received placebo tablets. Results: About one-fourth (23.6\%) of the patients in the intervention arm and onethird (31.6\%) in the placebo arm were tested reverse transcriptase polymerase chain reaction (RTPCR) negative for SARS-CoV-2 on $6^{\text {th }}$ day. Although this difference was found to be statistically insignificant [rate ratio (RR): 0.8; 95\% confidence interval (CI): 0.4-1.4; $\mathrm{p}=0.348$ ]. All patients in the ivermectin group were successfully discharged. In comparison the same for the placebo group was observed to be $93 \%$. This difference was found to be statistically significant (RR: $1.1 ; 95 \% \mathrm{CI} ; 1.0-1.2 ; \mathrm{p}=0.045$ ). Conclusions: Inclusion of ivermectin in treatment regimen of mild to moderate COVID-19 patients could not be said with certainty based on our study results as it had shown only marginal benefit in successful discharge from the hospital with no other observed benefits.
\end{abstract}

\section{INTRODUCTION}

Although several treatment options (i.e., hydroxychloroquine, tocilizumab, remdesivir, ritonavir, lopinavir, convalescent plasma etc.) have been explored to treat coronavirus 2 (SARS-CoV2) infection, none has been convincingly found to be effective except low dose steroids in patients requiring supplemental oxygen or mechanical ventilation (1-7). There has been a growing interest in the anti-parasitic drug, ivermectin ever since it was reported to have an in-vitro activity against SARS-CoV-2. Its anti-viral activity, particularly against RNA viruses, is believed to be through the inhibition of nuclear import of several host and viral proteins $(8-10)$. The drug is already in use for treatment of onchocerciasis, lymphatic filariasis, strongyloidiasis, cutaneous larva migrans and scabies. For most helminthic infestations it is administered as a single oral dose of $150-200$ $\mu \mathrm{g} / \mathrm{kg}$. The dose may be repeated after 24 hours in intestinal strongyloidiasis $(11,12)$. Considering use of ivermectin in treatment of COVID-19 patients, several randomised controlled trials (RCTs) documented its beneficial role either as an additional monotherapy or in combination with doxycycline irrespective of severity of the disease (13-15). COVID-19 patients treated with ivermectin reported to have decreased duration of symptoms, days to COVID negativity (DTN), lower viral load, mortality rate and higher $\mathrm{SPO}_{2} \%$, platelet count and improvement of other prognostic laboratory parameters (13-17). A metanalysis (18) and several prior observational studies (19-21) have also documented similar observations. Although a recent double-blind RCT reported no benefit of ivermectin as an additional monotherapy in mild COVID-19 patients (22). 
Despite availability of mainly speculative literature on beneficial role of ivermectin, no prior such data available in an Indian context. Moreover, most prior RCTs either used a 5-day oral regimen $(13,14)$ or a parenteral regimen $(17)$ of ivermectin. Other RCTs used either a single oral dose of ivermectin alone (16) or in combination with doxycycline (15). Therefore, the effect of a two consecutive day oral ivermectin regimen in COVID-19 is yet to be explored by any RCT. Thus, this RCT was designed to elicit efficacy of two consecutive day $12 \mathrm{mg}$ ivermectin enteral regimen among mild to moderate COVID-19 patients admitted in a COVID dedicated healthcare facility of eastern India.

\section{MATERIALS AND METHODS}

This was a double blind, parallel, randomised, placebo-controlled trial conducted in the All India Institute of Medical Sciences (AIIMS), Patna which is situated in the eastern part of India. AIIMS-Patna is an institute of national importance which served as a COVID dedicated tertiary healthcare facility during July-December 2020. The institute has state of the art COVID care facility with about 440 general beds and 60 intensive care unit (ICU) beds and over 3000 dedicated and trained healthcare staff.

All adult patients (aged $\geq 18$ years) admitted with a diagnosis of COVID-19 (on the basis of a positive reverse transcriptase polymerase chain reaction (RTPCR) or rapid antigen test report) at our hospital with mild or moderate disease on admission as defined by the Ministry of Health and Family Welfare (MOHFW), Government of India (GOI) guidelines (23) and not meeting any of the exclusion criteria were considered eligible for the study. The exclusion criteria used for the study were: known allergy or adverse drug reaction with ivermectin; unwillingness or inability to provide consent to participate in the study; prior use of ivermectin during the course of current illness; pregnancy and lactation.

Definitions of mild, moderate, and severe COVID-19 used during enrolment of the study participants were as following: Mild: No evidence of breathlessness or hypoxia (normal saturation) (23); Moderate: Breathlessness and/or hypoxia (saturation $90-94 \%$ on room air), respiratory rate of 24 or more and no features of severe disease (23); Severe: Any of the following - severe respiratory distress, oxygen saturation $<90 \%$ on room air, respiratory rate $>30$, shock or evidence of a lifethreatening organ dysfunction (23).

All COVID-19 cases admitted between $1^{\text {st }}$ August and $31^{\text {st }}$ October 2020, meeting the study eligibility criteria, and volunteered to participate were included in the trial. Overall, 115 study participants could be enrolled in the trial during the study period. We approached these patients within 24 hours of their admission. After obtaining informed written consent, they were randomly allocated to either treatment group A or group B in a 1:1 ratio. Block randomisation was done with variable random block sizes of 4, 6 and 8. A random allocation list of 120 patients was generated using the sealed envelope (an online block randomisation list generating software) (24) and kept with a third person (not a part of the investigation team) prior to the commencement of the trial. Once an eligible study participant has provided consent for the trial, the investigation team doctor used to contact the concerned third person having the random allocation list over telephone to know the treatment group (A/B) for that particular patient. One of these two groups was the intervention group, and the other was the placebo group. However, up until the analysis of the data, this information was confined to the pharmacist dispensing the tablets. After confirmation of the treatment group, the investigation team doctor used to indent 2 tablets designated for that particular group. Both these treatment groups received 2 tablets similar in size, shape, colour, odour, and packaging on subsequent days. On recruitment, baseline characteristics of the patient such as: age, sex, comorbidities, duration of symptoms and disease severity on admission were noted down. All patients received usual care and treatment by their respective treating teams abiding by the standard treatment guidelines laid out by the institute (25). In addition, patients in the intervention arm received ivermectin $12 \mathrm{mg}$ while the patients in the non-interventional arm received placebo tablets on day 1 and 2 after their enrolment. These tablets were consumed by the patients on direct supervision of the investigation team. All study participants were followed up on subsequent days of study drugs administration to document any related adverse events.

All enrolled patients were supposed to undergo RTPCR for COVID-19 on $6^{\text {th }}$ day post their enrolment in the study. Their symptom status (asymptomatic or still symptomatic) on Day 6 were also documented. Similarly discharge status on $10^{\text {th }}$ day post enrolment, treatment received, requirement of ICU support, invasive mechanical ventilation during hospital stay and final inhospital treatment outcome (death/ discharge) of the study participants were also observed in this trial. Patient discharge decisions were solely made by the respective treating teams as per the guidelines of the institute (25). The investigation 
team did not have control over this. The discharge criteria of the institute during the trial were ten days past the onset of symptoms and afebrile for three consecutive days or maintaining oxygen saturation $>94 \%$ without supplemental oxygen for four consecutive days.

Out of the total 115 enrolled trial participants, 57 were randomly assigned to the intervention arm while 58 were randomly assigned to the placebo arm. One patient in either arm was administered unblinded ivermectin tablet by the treating team on day 2, hence excluded. Additionally, one patient in the intervention arm could not be tracked from day 2 . So, all these were considered as lost to follow-up. Excluding these three patients, 55 patients in the intervention arm and 57 patients in the placebo arm were included in the final analysis. (Figure 1)

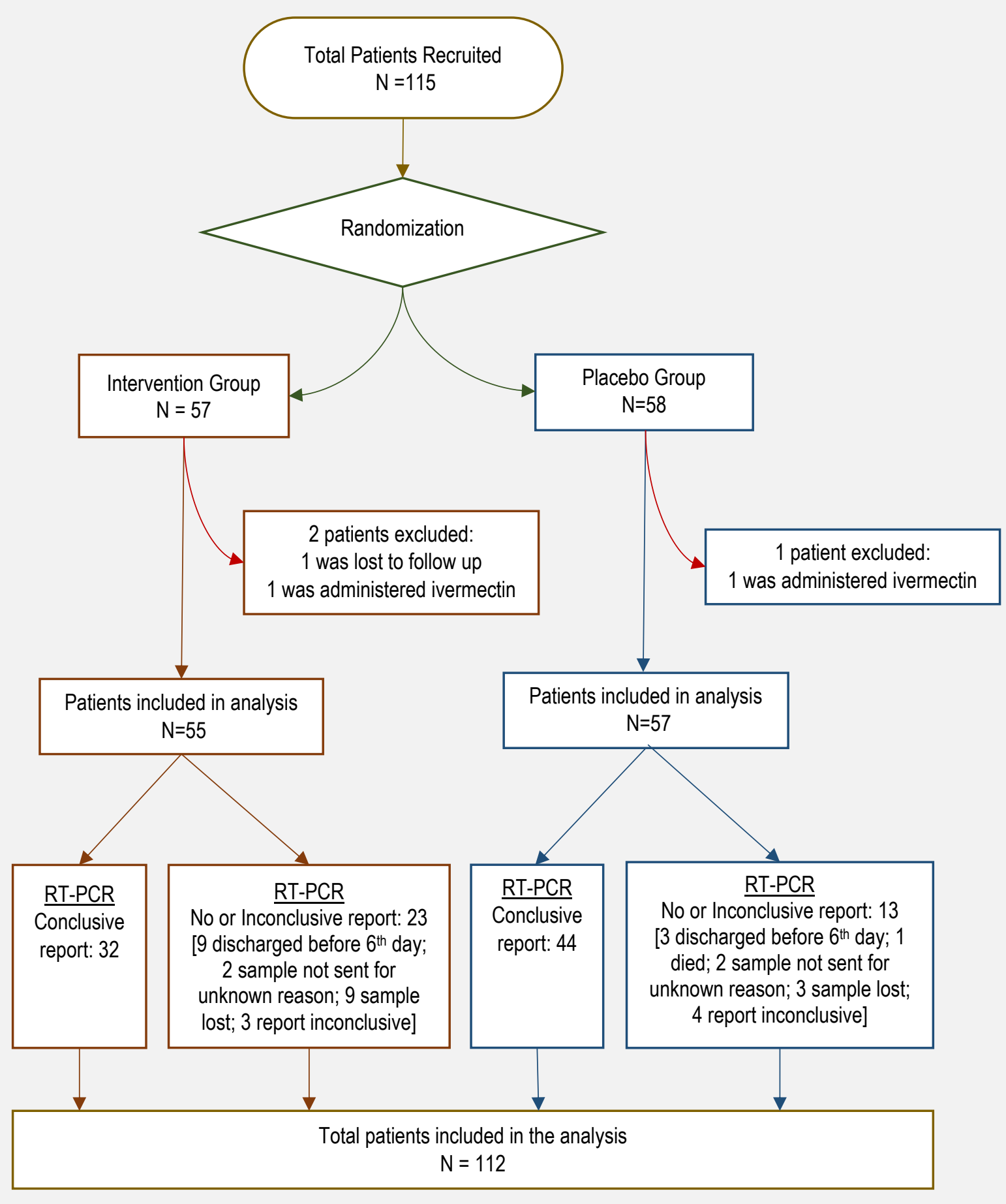

Figure 1. Flow-chart of the clinical trial showing recruitment of the study participants. 
The primary outcome of the trial was a negative RTPCR report on $6^{\text {th }}$ day. The secondary outcomes of the trial were symptom status on $6^{\text {th }}$ day, discharge status on $10^{\text {th }}$ day, requirement of ICU support, invasive mechanical ventilation and final treatment outcome (death / discharge) during hospital stay.

Statistical analysis was done using IBM Statistical Package for Social Sciences (SPSS) (Chicago, USA) software, version 22. Quantitative and qualitative variables documented for this study were reported in terms of mean (standard deviation) and frequency (percentage) respectively. To compare baseline characteristics of both the trial arms bivariate analysis was performed using the independent samples Student's t-test for continuous variables and chi square test for categorical variables. To compare various outcome measures of the study across interventional and non-interventional arms rate ratio (RR) was used. The minimum acceptable confidence level used for this trial was $\alpha=0.95$ and any observed difference with $\mathrm{p}<0.05$ was considered as statistically significant.

Ethical clearance was obtained from the Institutional Ethics Committee of AIIMS-Patna (Ref. No. - AIIMS/Pat/IEC/2020/521). The protocol of the trial was registered with the Clinical Trials Registry- India (CTRI) (registration number: CTRI/2020/08/027225). All these were done well before enrolment of the first study participant.

\section{RESULTS}

Baseline characteristics of the trial participants recruited for both ivermectin and placebo arms are depicted in Table 1. No significant difference was observed in terms of mean age, sex distribution, mean days since onset of symptoms, existing chronic co-morbidities, disease severity on admission and received treatment during hospital stay.

Table 1. Background Characteristics of the study subjects.

\begin{tabular}{|c|c|c|c|c|}
\hline Variable & $\begin{array}{c}\text { Ivermectin } \\
55(49.1 \%) \\
\text { n (\%) / } \\
\text { Mean } \pm \text { SD }\end{array}$ & $\begin{array}{c}\text { Placebo } \\
57(50.9 \%) \\
\text { n }(\%) / \\
\text { Mean } \pm \text { SD }\end{array}$ & $\begin{array}{c}\text { Total } \\
112(100 \%) \\
\text { n }(\%) / \\
\text { Mean } \pm \text { SD }\end{array}$ & $p$ \\
\hline Age in years: & $50.7 \pm 12.7$ & $54.2 \pm 16.3$ & $52.5 \pm 14.7$ & $0.218^{*}$ \\
\hline \multicolumn{5}{|l|}{ Sex: } \\
\hline Female & $15(27.3)$ & $16(28.1)$ & $31(27.7)$ & $0.925^{\#}$ \\
\hline Male & $40(72.7)$ & $41(71.9)$ & $81(72.3)$ & \\
\hline Days since onset of symptoms: & $6.1 \pm 3.6$ & $7.9 \pm 8.6$ & $6.9 \pm 6.6$ & $0.152^{*}$ \\
\hline \multicolumn{5}{|l|}{ Comorbidities: } \\
\hline Hypertension & $21(38.2)$ & $18(31.6)$ & $39(34.8)$ & $0.463^{\#}$ \\
\hline Diabetes & $21(38.2)$ & 19(33.3) & $40(35.7)$ & $0.592^{\#}$ \\
\hline Ischemic Heart Disease & $2(3.6)$ & $8(14.0)$ & $10(8.9)$ & $0.054^{\#}$ \\
\hline Heart Failure & $1(1.8)$ & $1(1.8)$ & $2(1.8)$ & $0.980^{\#}$ \\
\hline Chronic Kidney Disease & $1(1.8)$ & $2(3.5)$ & $3(2.7)$ & $0.580^{\#}$ \\
\hline Stroke & $0(0.0)$ & $0(0.0)$ & $0(0.0)$ & - \\
\hline Chronic Obstructive Pulmonary Disease & $1(1.8)$ & $0(0.0)$ & $1(0.9)$ & $0.306^{\#}$ \\
\hline Asthma & $1(1.8)$ & $0(0.0)$ & $1(0.9)$ & $0.306^{\#}$ \\
\hline Cancer & $2(3.6)$ & $4(7.0)$ & $6(5.4)$ & $0.427^{\#}$ \\
\hline Other comorbidities & $7(12.7)$ & $11(19.3)$ & $18(16.1)$ & $0.344^{\#}$ \\
\hline \multicolumn{5}{|l|}{ COVID-19 disease severity on admission: } \\
\hline Mild & $42(76.4)$ & $46(80.7)$ & $88(78.6)$ & $0.576^{\#}$ \\
\hline \multirow[t]{2}{*}{ Moderate } & $13(23.6)$ & $11(19.3)$ & $24(21.4)$ & \\
\hline & & & \multicolumn{2}{|c|}{ Table 1 continues ... } \\
\hline
\end{tabular}




\begin{tabular}{|c|c|c|c|c|}
\hline \multicolumn{5}{|l|}{ Treatments given: } \\
\hline Hydroxychloroquine & $55(100.0)$ & $57(100.0)$ & $112(100.0)$ & - \\
\hline Steroid & $55(100.0)$ & $57(100.0)$ & $112(100.0)$ & - \\
\hline Enoxaparin & $53(96.4)$ & $55(96.5)$ & $108(96.4)$ & $0.971^{\#}$ \\
\hline Antibiotics & $55(100.0)$ & $57(100.0)$ & $112(100.0)$ & - \\
\hline Remdesivir & $12(21.8)$ & $11(19.3)$ & $23(20.5)$ & $0.741^{\#}$ \\
\hline Convalescent Plasma & $8(14.5)$ & $7(12.3)$ & $15(13.4)$ & $0.725^{\#}$ \\
\hline Tocilizumab & $4(7.3)$ & $3(5.3)$ & $7(6.3)$ & $0.660^{\#}$ \\
\hline Other Drugs & $36(65.5)$ & $38(66.7)$ & $74(66.1)$ & $0.892^{\#}$ \\
\hline
\end{tabular}

"Independent samples Student's t test; " chi square test

About one-fourth $(23.6 \%)$ of the patients in the intervention arm and one-third (31.6\%) in the placebo arm were tested RTPCR negative for SARS-CoV-2 on $6^{\text {th }}$ day. The difference between the two groups was found to be statistically insignificant [RR: $0.8 ; 95 \%$ confidence interval (CI): 0.4-1.4; $\mathrm{p}=0.348]$. Considering resolution of symptoms on $6^{\text {th }}$ day, about four-fifth $(83.6 \%)$ of the patients in the intervention arm and nine-tenth $(89.5 \%)$ in the placebo arm were found to have achieved the same which was statistically indifferent (RR: 0.9; 95\% CI: 0.8-1.1; $\mathrm{p}=0.365$ ). Similarly, no statistical difference was observed in terms of discharge status on $10^{\text {th }}$ day $(80.0 \%$ in ivermectin group vs. $73.7 \%$ in placebo group) and ICU support requirement during hospital stay (9.1\% in ivermectin group vs. $10.5 \%$ in placebo group). Only $1.8 \%$ in the intervention arm needed invasive mechanical ventilation compared to $8.8 \%$ in the placebo arm. However, this difference was not statistically significant (RR: 0.2 ; 95\% CI: 0.0-
$1.7 ; \mathrm{p}=0.102)$. Mortality was found to be $7 \%(\mathrm{n}=4)$ in the placebo arm which was null for the intervention arm. Conversely, all patients in the ivermectin group were successfully discharged. In comparison the same for the placebo group was observed to be $93 \%$. This difference was found to be statistically significant (RR: $1.1 ; 95 \% \mathrm{CI} ; 1.0$ 1.2; $\mathrm{p}=0.045)$. Notably, no adverse events attributable to ivermectin were reported during this trial.

\section{DISCUSSION}

This was the first experimental study which elicited efficacy of two consecutive day enteral regimen of ivermectin in treatment of mild to moderate COVID-19. Third party random allocation, doubleblinding and no significant difference in background rates of the study participants of both the arms made the findings of the study more robust.

Table 2. Distribution of the study subjects according to the primary and secondary outcomes

\begin{tabular}{lcccc}
\hline Outcome Variable & $\begin{array}{c}\text { Ivermectin } \\
(\mathbf{n = 5 5}) \\
\text { Outcome } \mathbf{n}(\boldsymbol{\%})\end{array}$ & $\begin{array}{c}\text { Placebo } \\
(\mathbf{n = 5 7 )} \\
\text { Outcome } \mathbf{n}(\boldsymbol{\%})\end{array}$ & $\begin{array}{c}\text { Rate Ratio } \\
\mathbf{R R} \\
(\mathbf{9 5 \%} \mathbf{C I})\end{array}$ & $\boldsymbol{p}^{*}$ \\
\hline $\begin{array}{l}\text { Primary Outcome } \\
\text { Negative RT-PCR on day 6 }\end{array}$ & $13(23.6)$ & $18(31.6)$ & $0.8(0.4-1.4)$ & 0.348 \\
Secondary Outcomes & & & & \\
Symptom free on day 6 & $46(83.6)$ & $51(89.5)$ & $0.9(0.8-1.1)$ & 0.365 \\
Discharged by day 10 & $44(80.0)$ & $42(73.7)$ & $1.1(0.9-1.3)$ & 0.429 \\
Admission to ICU & $5(9.1)$ & $6(10.5)$ & $0.9(0.3-2.7)$ & 0.799 \\
Invasive Ventilation & $1(1.8)$ & $5(8.8))$ & $0.2(0.0-1.7)$ & 0.102 \\
Final outcome: discharge & $55(100.0)$ & $53(93.0)$ & $1.1(1.0-1.2)$ & 0.045 \\
Final outcome: in-hospital mortality & $0(0)$ & $4(7)$ & 0 & - \\
\hline
\end{tabular}

RR: Rate Ratio, CI: confidence interval; ${ }^{*}$ chi square test 
In the present study no significant difference was documented in terms of $6^{\text {th }}$ day negative RTPCR for SARS-CoV-2. Rather probability of this positive outcome among participants in the intervention arm $(23.6 \%)$ was lower compared to the placebo arm (31.6\%). This might be attributable to higher inconclusive or missing $6^{\text {th }}$ day RTPCR report in the intervention arm $(41.8 \%)$ compared to the placebo arm (22.8\%). Mahmud et al. (15) found lower $14^{\text {th }}$ day RTPCR positivity rate in intervention group (7.6\%) (received tablet ivermectin $12 \mathrm{mg}$ on day 1 and tablet doxycycline $100 \mathrm{mg}$ twice daily for 5 days) compared to the placebo group $(20.0 \%)$ which was unlike our observations. However, due to the use of different intervention and day of RTPCR testing, results of Mahmud et al. (15) and our study is not completely comparable. Moreover, it has been suggested that prolonged viral RNA shedding may continue for weeks after recovery leading to a false positive RTPCR report even though the patient may not be infectious anymore (26). Thus, it is questionable whether a negative repeat RTPCR is a clinically useful outcome measure for COVID-19.

In terms of symptom resolution on $6^{\text {th }}$ day, we observed no difference between ivermectin and placebo group. A recently published large RCT on 400 mild COVID-19 patients did not observed any difference with a five-day course of oral ivermectin $(300 \mu \mathrm{g} / \mathrm{kg})$ in terms of time to resolution of symptoms and $21^{\text {st }}$ day symptom resolution compared to placebo (22). This was consistent with the findings of the current study. Although, contrary findings were reported by another large RCT by Mahmud et al. (15). In that study patients recruited in the intervention arm had higher 7-day recovery $(61.0 \%)$ compared to the placebo arm (44.0\%). However, the overall $6^{\text {th }}$ day recovery rate was higher in both the arms in the present study compared to $7^{\text {th }}$ day recovery rates reported by Mahmud et al. (15). Moreover, Mahmud et al. (15) used both ivermectin and doxycycline (oral $12 \mathrm{mg}$ ivermectin on day 1 and doxycycline $100 \mathrm{mg}$ twice daily for 5 days) in the intervention arm which was unlike us. Tenth day discharge was marginally higher in ivermectin group $(80.0 \%)$ compared to the placebo group (73.7\%). Although this difference was not statistically significant. This might have been influenced by $10^{\text {th }}$ day discharge rule set by our institute to accommodate as many COVID-19 patients of the region as possible. AIIMS-Patna is the highest referral institute in the state of Bihar for not only COVID-19 but for any medical or surgical condition owing to its state-ofthe-art facilities and trained healthcare staff. An Iranian RCT by Shahbaznejad et al. (27) reported significantly lower duration of hospital stay [median \pm standard error (SE): $6.0 \pm 0.5$ days)] in patients treated with single enteral dose of ivermectin compared to the placebo group [Median \pm SE: $7.0 \pm 0.4$ days)] which was discordant to our observations. ICU support requirement were found to be similar in both the groups $(9.1 \%$ in ivermectin group vs. $10.5 \%$ in placebo group) in the present study. Although in terms of mechanical ventilation requirement, ivermectin group done better (1.8\%) compared to the placebo group (8.8\%). This association needs be explored further by the future RCTs with a considerably larger sample size to remark upon something with certainty. The only significant positive outcome of the current study was a higher rate of successful discharge in the ivermectin group compared to the placebo group; although the difference found was quite marginal. This was also noted in some prior observational studies conducted by Khan et al. (21) and Rajter et al. (28). The RCT conducted by Mahmoud et al. (15) has reported lower mortality rate in intervention arm (ivermectin and doxycycline) (0.0\%) compared to the placebo group (1.7\%) (15). This was somewhat similar to our observations.

The findings of this trial should be interpreted with consideration of certain limitations. Firstly, absence of conclusive $6^{\text {th }}$ day RTPCR report in $32.1 \%$ of the cases $(41.8 \%$ in intervention arm and $22.8 \%$ in placebo arm) diluted our primary trial outcome. Secondly, as serial RTPCR tests could not be considered due to feasibility, the median time to viral clearance in the two groups could not be ascertained.

\section{CONCLUSION}

Inclusion of ivermectin in treatment regimen of mild to moderate COVID-19 patients could not be recommended with certainty based on our study results as it had shown only marginal benefit in successful discharge from the hospital with no other observed benefits. Larger, multicentre RCTs should be planned to provide a clearer answer.

\section{ACKNOWLEDGEMENTS.}

We would like to thank Dr Prabhat Kumar Singh, Director AIIMS Patna for facilitating the study, Dr. Alok Ranjan, Assistant Professor, Department of Community and Family Medicine, AIIMS, Patna for reviewing the statistical analysis, Mr Sunil Verma of Sun Pharma Pvt. Ltd. for arranging the placebo tablets and Mr Ambuj Kumar, Pharmacist at AIIMS, Patna for dispensing the tablets and ensuring blinding. 
FINANCIAL DISCLOSURE. Prior permission was obtained from AIIMS, Patna administration for repeat RT-PCR tests (Project Code 521, Letter no. RC/AIIMS/Pat/2020/49). Ivermectin tablets were procured from the learning resource allowance of the principal investigator. Placebo tablets were provided by Sun Pharma Pvt. Ltd.

\section{CONFLICT OF INTEREST. None}

INFORMED CONSENT. Informed written consent was obtained from each study participant before their enrolment.

\section{AUTHOR CONTRIBUTIONS.}

Conceptualization: Ravikirti, Neeraj Agarwal, Bijit Biswas; Data curation: Ranjini Roy, Chandrima Pattadar, Rishav Raj, Bijit Biswas, Pramod Kumar Manjhi, Deependra Kumar Rai, Shyama, Anjani Kumar, Asim Sarfaraz; Formal analysis: Bijit Biswas, Ravikirti, Neeraj Agarwal. Funding acquisition: Ravikirti; Methodology: Ravikirti, Bijit Biswas, Neeraj Agarwal, Pramod Kumar Manjhi, Deependra Kumar Rai, Shyama, Anjani Kumar, Asim Sarfaraz; Project administration: Ravikirti, Ranjini Roy, Chandrima Pattadar, Rishav Raj, Bijit Biswas, Pramod Kumar Manjhi, Deependra Kumar Rai, Shyama, Anjani Kumar, Asim Sarfaraz; Visualization: Ravikirti, Bijit Biswas, Neeraj Agarwal; Writing - original draft: Ravikirti, Ranjini Roy, Chandrima Pattadar, Rishav Raj, Bijit Biswas; Writing - review \& editing: Ravikirti, Ranjini Roy, Chandrima Pattadar, Rishav Raj, Bijit Biswas, Neeraj Agarwal, Pramod Kumar Manjhi, Deependra Kumar Rai, Shyama, Anjani Kumar, Asim Sarfaraz.

\section{INSTITUTIONAL REVIEW BOARD APPROVAL. Ethical clearance was obtained from the Institutional Ethics Committee of AIIMS- Patna (Ref. No. - AIIMS/Pat/IEC/2020/521).}

\section{ETHICAL COMPLIANCE WITH} HUMAN/ANIMAL STUDY. This study was conducted in compliance with the ethical standards of the responsible institution on human subjects as well as with the Helsinki Declaration.

DATA AVAILABILITY. Inquiries should be directed to the corresponding author.

\section{REFERENCES:}

1. Horby P, Lim WS, Emberson JR, et al. Dexamethasone in Hospitalized Patients with Covid-19. $N$ Engl $J$ Med. 2021;384(8):693-704. doi:10.1056/NEJMoa2021436.

2. Sterne JAC, Murthy S, Diaz JV, et al. Association Between Administration of Systemic Corticosteroids and Mortality Among Critically Ill Patients With COVID19: A Meta-analysis. JAMA. 2020;324(13):1330-1341. doi:10.1001/jama.2020.17023.

3. Abani O, Abbas A, Abbas F, et al. Tocilizumab in patients admitted to hospital with COVID-19 (RECOVERY): a randomised, controlled, open-label, platform trial. Lancet Lond Engl. 2021;397(10285):1637-1645. doi:10.1016/S0140-6736(21)00676-0.

4. Beigel JH, Tomashek KM, Dodd LE, et al. Remdesivir for the Treatment of Covid-19 Final Report. $N$ Engl $J$ Med. 2020;383(19):1813-1826. doi:10.1056/NEJMoa2007764.

5. Agarwal A, Mukherjee A, Kumar G, et al. Convalescent plasma in the management of moderate covid-19 in adults in India: open label phase II multicentre randomised controlled trial (PLACID Trial). BMJ. 2020;371:m3939. doi:10.1136/bmj.m3939.

6. Cao B, Wang Y, Wen D, et al. A Trial of Lopinavir-Ritonavir in Adults Hospitalized with Severe Covid-19. N Engl J Med. 2020;382(19):1787-1799.

doi:10.1056/NEJMoa2001282.

7. Md Insiat Islam Rabby. Current Drugs with Potential for Treatment of COVID-19: A Literature Review. J Pharm Pharm Sci. 2020;23(1):58-64. doi:10.18433/jpps31002

8. Caly L, Druce JD, Catton MG, Jans DA, Wagstaff KM. The FDA-approved drug ivermectin inhibits the replication of SARSCoV-2 in vitro. Antiviral Res. 2020;178:104787.

doi:10.1016/j.antiviral.2020.104787.

9. Heidary F, Gharebaghi R. Ivermectin: a systematic review from antiviral effects to COVID-19 complementary regimen. $J$ Antibiot (Tokyo). 2020;73(9):593-602. doi:10.1038/s41429-020-0336-z.

10. Kinobe RT, Owens L. A systematic review of experimental evidence for antiviral effects of ivermectin and an in silico analysis of ivermectin's possible mode of action against SARS-CoV-2. Fundam Clin Pharmacol. 2021;35(2):260-276. doi:10.1111/fcp. 12644 . 
11. Gonzalez P, A. Gonzalez F, Ueno K. Ivermectin in Human Medicine, An Overview of the Current Status of Its Clinical Applications. Curr Pharm Biotechnol. 2012;13(6):1103-1109. doi:10.2174/138920112800399248.

12. Ashour DS. Ivermectin: From theory to clinical application. Int J Antimicrob Agents. 2019;54(2):134-142.

doi:10.1016/j.ijantimicag.2019.05.003.

13. Ahmed S, Karim MM, Ross AG, et al. A five-day course of ivermectin for the treatment of COVID-19 may reduce the duration of illness. Int J Infect Dis IJID Off Publ Int Soc Infect Dis. 2021;103:214-216. doi:10.1016/j.ijid.2020.11.191.

14. Okumuş N, Demirtürk N, Çetinkaya RA, et al. Evaluation of the effectiveness and safety of adding ivermectin to treatment in severe COVID-19 patients. BMC Infect Dis. 2021;21(1):411. doi:10.1186/s12879-02106104-9.

15. Mahmud R, Rahman MM, Alam I, et al. Ivermectin in combination with doxycycline for treating COVID-19 symptoms: a randomized trial. $J$ Int Med Res. 2021;49(5):3000605211013550. doi:10.1177/03000605211013550.

16. Chaccour C, Casellas A, Blanco-Di Matteo A, et al. The effect of early treatment with ivermectin on viral load, symptoms and humoral response in patients with nonsevere COVID-19: A pilot, double-blind, placebo-controlled, randomized clinical trial. EClinicalMedicine. 2021;32:100720. doi:10.1016/j.eclinm.2020.100720.

17. Babalola OE, Bode CO, Ajayi AA, et al. Ivermectin shows clinical benefits in mild to moderate COVID19: a randomized controlled double-blind, dose-response study in Lagos. QJM Int J Med. Published online February 18, 2021:hcab035. doi:10.1093/qjmed/hcab035.

18. Padhy BM, Mohanty RR, Das S, Meher BR. Therapeutic potential of ivermectin as add on treatment in COVID 19: A systematic review and meta-analysis. J Pharm Pharm Sci. doi:10.18433/jpps31457.

19. Alam MT, Murshed R, Bhiuyan E, Saber S, Alam RF, Robin RC. A Case Series of 100 COVID-19 Positive Patients Treated with Combination of Ivermectin and Doxycycline. J Bangladesh Coll Physicians Surg. 2020;38(COVID-19 (Supplement Issue)):10-15. doi:10.3329/jbcps.v38i0.47512.
20. Bhattacharya R, Ray I, Mukherjee R, Chowdhury S, Kulasreshtha M, Ghosh R. Observational Study on Clinical Features, Treatment and Outcome of COVID 19 In A Tertiary Care Centre in India- A Retrospective Case Series. Int J Sci Res. 2020;9(10):69-71. doi:10.36106/ijsr/7232245.

21. Khan MSI, Khan MSI, Debnath CR, et al. Ivermectin Treatment May Improve the Prognosis of Patients With COVID-19. Arch Bronconeumol. 2020;56(12):828-830. doi:10.1016/j.arbres.2020.08.007.

22. López-Medina E, López P, Hurtado IC, et al. Effect of Ivermectin on Time to Resolution of Symptoms Among Adults With Mild COVID-19: A Randomized Clinical Trial. JAMA. 2021;325(14):1426-1435. doi:10.1001/jama.2021.3071.

23. Clinical Management Protocol: COVID-19 (Version 3). Published online June 13, 2020. https://www.mohfw.gov.in/pdf/ClinicalMan agementProtocolforCOVID19.pdf.

24. Create a blocked randomisation list | Sealed Envelope. Accessed May 26, 2021. https://www.sealedenvelope.com/simplerandomiser/v1/lists.

25. Standard Operating Procedure \& Hand Book of COVID-19:Management (Version 3). Published online January 7, 2020. Accessed December 24, 2020. https://aiimspatna.edu.in/advertisement/Cov id_SOP_AIIMS_P_version3.0.pdf.

26. Surkova E, Nikolayevskyy V, Drobniewski F. False-positive COVID-19 results: hidden problems and costs. Lancet Respir Med. 2020;8(12):1167-1168. doi:10.1016/S22132600(20)30453-7.

27. Shahbaznejad L, Davoudi A, Eslami G, et al. Effect of ivermectin on COVID-19: A multicenter double-blind randomized controlled clinical trial. Clin Ther. Published online May 2021:S0149291821002010. doi:10.1016/j.clinthera.2021.04.007.

28. Rajter JC, Sherman MS, Fatteh N, Vogel F, Sacks J, Rajter JJ. Use of Ivermectin Is Associated With Lower Mortality in Hospitalized Patients With Coronavirus Disease 2019: The Ivermectin in COVID Nineteen Study. Chest. 2021;159(1):85-92. doi:10.1016/j.chest.2020.10.009. 